\title{
Structurally driven magnetic state transition of biatomic Fe chains on $\operatorname{Ir}(001)$
}

\author{
Yuriy Mokrousov, ${ }^{1,2, *}$ Alexander Thiess, ${ }^{1,2}$ and Stefan Heinze ${ }^{1,3}$ \\ ${ }^{1}$ Institute of Applied Physics, University of Hamburg, D-20355 Hamburg, Germany \\ ${ }^{2}$ Institut für Festkörperforschung and Institute for Advanced Simulation, Forschungszentrum Jülich, D-52425 Jülich, Germany \\ ${ }^{3}$ Institute of Theoretical Physics and Astrophysics, Christian-Albrechts-University of Kiel, D-24098 Kiel, Germany
}

(Received 29 June 2009; revised manuscript received 3 September 2009; published 24 November 2009)

\begin{abstract}
Using first-principles calculations, we demonstrate that the magnetic exchange interaction and the magnetocrystalline anisotropy of biatomic Fe chains grown in the trenches of the $(5 \times 1)$ reconstructed $\operatorname{Ir}(001)$ surface depend sensitively on the atomic arrangement of the Fe atoms. Two structural configurations have been considered which are suggested from recent experiments. They differ by the local symmetry and the spacing between the two strands of the biatomic Fe chain. Since both configurations are very close in total energy they may coexist in experiment. We have investigated collinear ferro- and antiferromagnetic solutions as well as a collinear state with two moments in one direction and one in the opposite direction $(\uparrow \downarrow \uparrow-s t a t e)$. For the structure with a small interchain spacing, there is a strong exchange interaction between the strands and the ferromagnetic state is energetically favorable. In the structure with larger spacing, the two strands are magnetically nearly decoupled and exhibit antiferromagnetic order along the chain. In both cases, due to hybridization with the Ir substrate the exchange interaction along the chain axis is relatively small compared to free-standing biatomic iron chains. The easy magnetization axis of the Fe chains also switches with the structural configuration and is out-of-plane for the ferromagnetic chains with small spacing and along the chain axis for the antiferromagnetic chains with large spacing between the two strands. Calculated scanning tunneling microscopy images and spectra suggest the possibility to experimentally distinguish between the two structural and magnetic configurations.
\end{abstract}

DOI: 10.1103/PhysRevB.80.195420

PACS number(s): 75.75.+a

\section{INTRODUCTION}

Driven by the wish to realize the proposed concepts of future spintronic devices ${ }^{1-3}$ the development of novel nanostructures and nanomaterials with tailored electronic and magnetic properties has become a key challenge of today's research. A promising path to control the magnetic properties of matter is to use low-dimensional systems and to reduce their size down to the nanometer or even atomic scale. For nanoscale systems, however, an essential requirement is to enhance the magnetic anisotropy in order to stabilize magnetic order against thermal fluctuations or quantum tunneling. The manipulation of exchange interactions opens another path to create new materials with a magnetic state that may be tunable by external magnetic or electric fields. ${ }^{4}$ E.g., the occurrence of chiral spin spiral states at surfaces has been demonstrated, ${ }^{5,6}$ their manipulation by electrical currents was suggested $^{5,7}$ and a way to grow films with multiple metastable magnetic states has been proposed. ${ }^{8}$

The ability to create one-dimensional (1D) monoatomic magnetic chains of transition metals on surfaces by self-organization ${ }^{9,10}$ or by manipulation with a scanning tunneling microscope ${ }^{11}$ has recently opened new vistas to explore and manipulate artificial magnetic nanostructures even atom by atom. E.g. the pioneering work of Gambardella et $a l .{ }^{9,10}$ demonstrated that the magnetic anisotropy of atomic transition-metal chains, consisting of Co atoms on a stepped $\mathrm{Pt}(111)$ surface, is dramatically enhanced with decreasing dimensionality from two-dimensional films to quasi-onedimensional chains and depends sensitively on the number of Co strands in a chain. These experimental observations were explained based on electronic structure calculations which emphasized the crucial role played by the substrate, reduced symmetry, and structural relaxations for the magnetocrystalline anisotropy. ${ }^{12-15}$ The large magnetic anisotropies led to slow relaxation dynamics of the magnetization and the observation of magnetic hysteresis loops at low temperatures indicative of ferromagnetic coupling. In another experiment, Mn chains of up to 10 atoms were created by manipulation with a scanning tunneling microscopy tip on an insulating $\mathrm{CuN}$ layer grown on $\mathrm{Cu}(001)$. Experimentally, the exchange interaction between individual spins was obtained by measuring the excitation spectrum via inelastic tunneling spectroscopy which showed the quantum behavior of the entire chain. ${ }^{11}$ Even the sign and size of the exchange interaction between the $\mathrm{Mn}$ atoms could be extracted from the experimental data. Calculations based on density-functional theory (DFT) clarified that a superexchange mechanism along the Mn-N-Mn bond is responsible for the weak antiferromagnetic coupling. ${ }^{16,17}$

Recently, Hammer et al. have used a combination of IVLEED (low-energy electron diffraction) and scanning tunneling microscopy (STM) measurements to demonstrate that the $\operatorname{Ir}(001)$ surface can serve as an ideal template to grow defectfree nanometer-long transition-metal nanowires of different structure, chemical composition, and length depending on the preparation conditions. ${ }^{18-20}$ E.g., biatomic Fe chains can be created on the $(5 \times 1)$ reconstructed $\operatorname{Ir}(001)$ surface and lifting the surface reconstruction by hydrogen opens the possibility to produce Fe-Ir-Fe triatomic chains. While the biatomic Fe chains are a very promising system to study magnetism of (quasi-)one-dimensional transition-metal chains, there is little understanding so far. Experimentally, it is extremely challenging as for laterally averaging measurements samples with a homogeneous distribution of chains are 

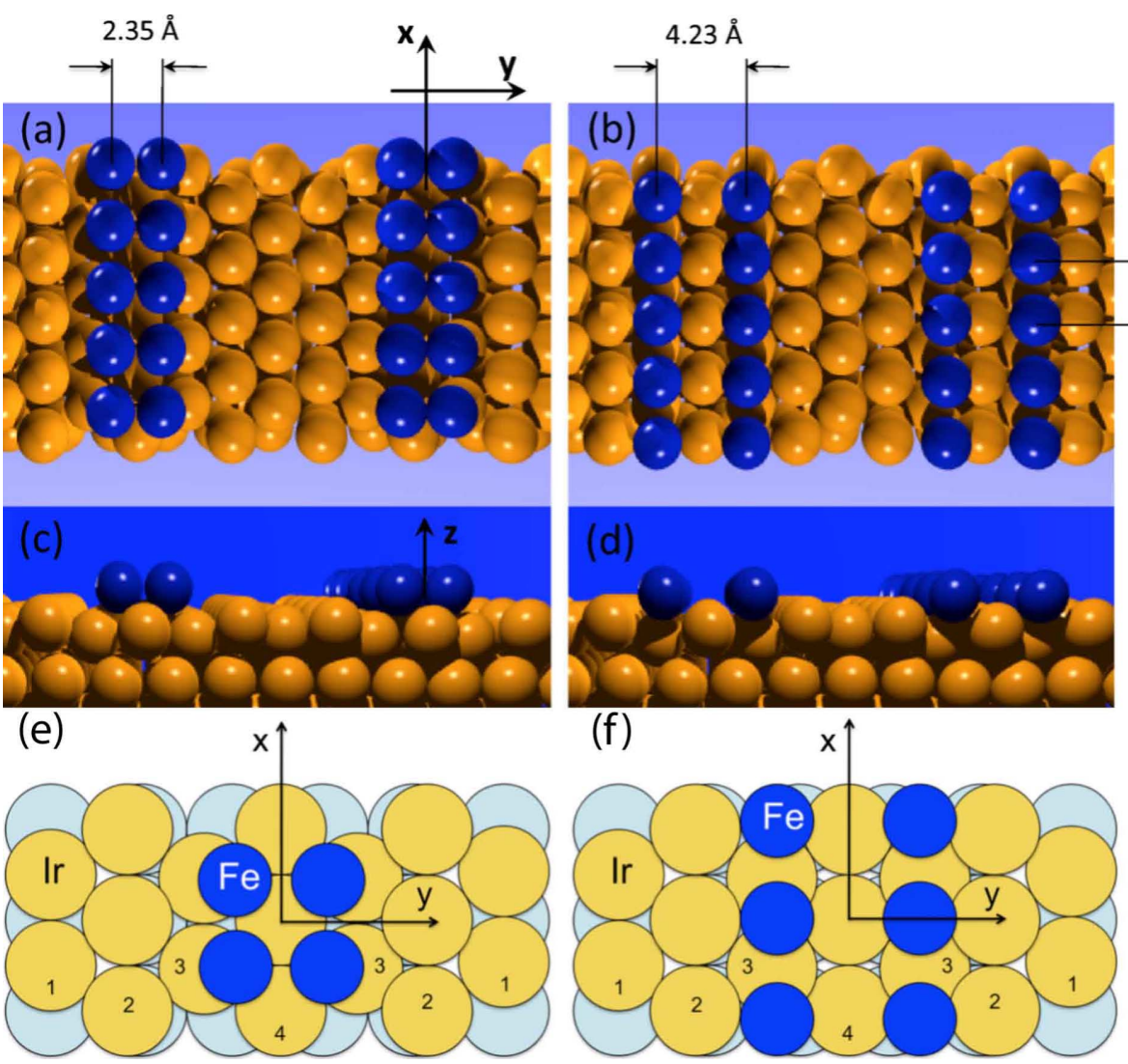

FIG. 1. (Color online) Geometrical structure of the biatomic $\mathrm{Fe}$ chains on the $(5 \times 1)$ reconstructed $\operatorname{Ir}(001)$ substrate in $\mathrm{C} 1$ [(a) top view; (c) side view] and C4 [(b) top view; (d) side view] configurations. $\mathrm{Fe}$ atoms are marked in blue, while Ir atoms are marked in gold. In (e) and (f) a schematic top view of, respectively, $\mathrm{C} 1$ and $\mathrm{C} 4$ biatomic $\mathrm{Fe}$ chains is shown (positions of the atoms do not correspond to realistic calculated values). In the latter graphs the numbering of the Ir atoms corresponds to that in Fig. 2 and Table II. The $x$ and $y$ axes defined in (a) correspond to the [011] and [0 $\overline{1} 1]$ directions, respectively. needed or a technique must be applied which allows to locally probe the magnetic properties of individual Fe chains. Another key difficulty is the detailed characterization of the chains structure. From combined STM and LEED experiments it is only known that the biatomic chains grow in the trenches of the $(5 \times 1)$ reconstructed $\operatorname{Ir}(001)$ surface, however, the adsorption sites in the trenches could not be deduced. As structure and magnetism are closely correlated in such systems, their magnetic properties are an open issue.

A theoretical study using first-principles calculations ${ }^{21}$ reported an excellent agreement with the structural parameters of the $(5 \times 1)$ reconstructed $\operatorname{Ir}(001)$ surface and concluded that the Fe chains are strongly ferromagnetic at low temperatures but were probably nonmagnetic (NM) in the roomtemperature measurements of Hammer et al. However, this theoretical study considered only ferromagnetic solutions and did not determine the magnetocrystalline anisotropy energy which is crucial for an experimental verification of the proposed ferromagnetism in these chains. On the other hand, a $5 d$ transition-metal substrate such as Ir can have dramatic consequences on the exchange coupling in a deposited $\mathrm{Fe}$ nanostructure as is apparent from the observation of a complex nanoscale magnetic structure for an Fe monolayer on $\operatorname{Ir}(111),{ }^{22}$ the antiferromagnetic ground state of an Fe monolayer on W(001), ${ }^{23}$ to name just a few. Recent studies on $\mathrm{Fe}$ stripes on $\mathrm{Pt}(997)$ (Ref. 24) and FePt surface alloys ${ }^{25}$ report on strong correlation between complex magnetic ground states and the details of structural arrangement.

Here, we use first-principles calculations based on density-functional theory to study the structural, electronic, and magnetic properties of biatomic Fe chains deposited in the trenches of the $(5 \times 1)$ reconstructed $\operatorname{Ir}(001)$ surface. $^{26}$ We focus on two structural arrangements of the biatomic chains which differ by the adsorption sites of the Fe atoms. In one configuration the distance between the two strands of the biatomic chain is smaller than the atom spacing along the chain direction (denoted as C1 in accordance with Ref. 21), while in the other their separation is clearly larger than the interchain spacing (denoted as C4); see Fig. 1. We consider collinear ferromagnetic (FM) and antiferromagnetic (AFM) arrangements along the chains and the $\uparrow \downarrow \uparrow$ state with two moments in one and one moment in the opposite direction. We find that the energetically favorable magnetic state as well as the easy magnetization axis of the Fe chains depend sensitively on their atomic arrangement and local symmetry. For the Fe chains in the $\mathrm{C} 1$ structure, the exchange coupling along the chain is ferromagnetic; however, due to hybridization with the Ir substrate it is much weaker than for freestanding biatomic Fe chains with the same atom spacing. Due to their small separation, the two strands of the C1 chains are also ferromagnetically coupled. Surprisingly, for the $\mathrm{C} 4$ configuration, we find a transition from ferromagnetic state for free-standing chains to antiferromagnetic order along the biatomic chains after deposition on the Ir substrate. In this case, the hybridization with the Ir surface is strong enough to invert the sign of exchange coupling, while the two Fe strands are nearly exchange decoupled. The interplay of the Fe interstrand distance and the hybridization with the Ir substrate results also in a different easy axis of the magnetization for the two structures: while in the C1-FM state the easy axis is out-of-plane, it switches into the chain axis for the C4-AFM configuration. The total energy of the $\mathrm{C} 1$ 
and C4 structures are quite close and therefore both chain types could occur in an experiment depending on the growth conditions. We simulate measurements by STM and observe that the two strands of the biatomic Fe chain in the C1ferromagnetic state are too close to be individually resolved, while they can be distinguished in the $\mathrm{C} 4$ structure with an antiferromagnetic ground state. In the latter case, spinpolarized STM (SP-STM) should further allow to directly resolve the twofold magnetic periodicity along the chain.

The structure of this paper is as follows. In the next section we present details of our method and the calculations. Then we discuss the structural relaxations of the pure $(5 \times 1)$ reconstructed $\operatorname{Ir}(001)$ substrate and of the biatomic $\mathrm{Fe}$ chains in the two structural configurations on the $(5 \times 1)$ reconstructed $\operatorname{Ir}(001)$ surface. In Sec. IV, we analyze the magnetic ground-state configuration and the effects of hybridization with the Ir substrate, before we turn to the magnetocrystalline anisotropy in Sec. V. We explore the feasibility to experimentally resolve the different structural and magnetic properties by SP-STM and to verify our predictions of a structure-dependent magnetic ground state. Finally, a conclusion and summary is given.

\section{COMPUTATIONAL DETAILS}

We employed the film version of the full-potential linearized augmented plane-wave (FLAPW) method, as implemented in the Jülich DFT code FLEUR. We used inversionsymmetric films with seven and nine layers of the $(5 \times 1)$ reconstructed $\operatorname{Ir}(001)$ surface and Fe biatomic chains on both sides of the slab. The whole system possesses spatial inversion symmetry. We calculate the biatomic Fe chains in a $(5$ $\times 1)$ supercell along the $y$ direction (the [011] axis), perpendicular to the chain $x$ axis (the [011] axis), which results in a distance of $13.75 \AA$ between the axes of two adjacent biatomic chains (cf. Fig. 1 for structural arrangements and definition of the axes). The theoretical Ir lattice constant we used for calculations constituted a value of $3.89 \AA$. We used the generalized-gradient approximation (GGA, revPBE functional $^{27}$ ) of the exchange-correlation potential for the structural relaxations and tested total energy differences between the two magnetic configurations also within the localdensity approximation (LDA, VWN functional ${ }^{28}$ ). We used $18 k$ points in a quarter of the full two-dimensional Brillouin zone (2D-BZ) for self-consistent calculations. The calculated total energy differences between different magnetic ground states were carefully tested with respect to the number of $k$ points. For the basis functions, we used a cutoff parameter of $k_{\max }=3.6$ a.u. $^{-1}$ for relaxations and 3.7 a.u. ${ }^{-1}$ for comparing the total energies of different magnetic configurations.

We considered two possible structural arrangements of the Fe atoms denoted as $\mathrm{C} 1$ and $\mathrm{C} 4$ according to the notation of Ref. 21, which are shown in Fig. 1. Experimentally, it has been observed by STM (Ref. 18) that the biatomic chains grow in the trenches of the $(5 \times 1)$ reconstructed $\operatorname{Ir}(001)$ surface and we therefore focus on these two configurations. Relaxations were performed until the forces changed by less than $3 \times 10^{-4} \mathrm{htr} / \mathrm{a} . \mathrm{u}$. The convergence of the relaxed atomic positions was carefully tested with respect to the
TABLE I. Relaxations of the Fe biatomic chains and the uppermost layer of the $(5 \times 1)$ reconstructed bare $\operatorname{Ir}(001)$ substrate for nine- and seven-layer slabs. All values are given in Ångstrom. The distances $d, b$, and $p$ correspond to those in Ref. 29 and are depicted in Fig. 2. For comparison the relaxations of the bare $\operatorname{Ir}(001)$ substrate from Ref. 29 (IV-LEED data) and Ref. 21 are given. For the interchain distance $\Delta$ values is brackets correspond to those calculated in Ref. 21.

\begin{tabular}{|c|c|c|c|c|c|}
\hline \multirow[b]{2}{*}{ Nine layers } & LEED & $(5 \times 1) \mathrm{Ir}$ & \multirow[b]{2}{*}{$(5 \times 1) \mathrm{Ir}$} & \multirow[b]{2}{*}{ C1-FM } & \multirow[b]{2}{*}{ C4-FM } \\
\hline & Ref. 29 & Ref. 21 & & & \\
\hline \multirow[t]{2}{*}{$\Delta$} & & & & 2.35 & 4.23 \\
\hline & & & & $(2.65)$ & $(4.35)$ \\
\hline$\delta$ & & & & 1.90 & 1.53 \\
\hline$d_{12}$ & 1.94 & 2.00 & 1.97 & 1.93 & 1.82 \\
\hline$b_{13}$ & 0.25 & 0.20 & 0.20 & 0.22 & 0.42 \\
\hline$b_{23}$ & 0.55 & 0.47 & 0.54 & 0.62 & 0.77 \\
\hline$b_{34}$ & 0.20 & 0.17 & 0.18 & 0.30 & 0.55 \\
\hline$p_{2}$ & 0.05 & 0.03 & 0.04 & 0.07 & 0.04 \\
\hline$p_{3}$ & 0.07 & 0.07 & 0.07 & 0.12 & 0.11 \\
\hline
\end{tabular}

Seven layers

\begin{tabular}{llll}
\hline$\Delta$ & & 2.40 & 4.19 \\
$\delta$ & & 1.90 & 1.54 \\
$d_{12}$ & 1.94 & 1.93 & 1.81 \\
$b_{13}$ & 0.25 & 0.22 & 0.42 \\
$b_{23}$ & 0.55 & 0.63 & 0.79 \\
$b_{34}$ & 0.20 & 0.30 & 0.53 \\
$p_{2}$ & 0.05 & 0.07 & 0.05 \\
$p_{3}$ & 0.07 & 0.13 & 0.12 \\
\hline \hline
\end{tabular}

computational parameters. Relaxations were performed only for the ferromagnetic state of both $\mathrm{C} 1$ and $\mathrm{C} 4$ configurations and the antiferromagnetic and the $\uparrow \downarrow \uparrow$ states were calculated on these atomic positions.

\section{STRUCTURE AND RELAXATIONS}

As a first step, we performed a structural relaxation of the pure $(5 \times 1)$ reconstructed $\operatorname{Ir}(001)$ surface. As can be seen in Table I, the results we obtain with a film of nine layers agree very well with the experimental data measured by IV-LEED. $^{29}$ In particular, the experimentally observed trenchlike structure is reproduced. Our values are also in close agreement with those obtained with the VASP code by Spišák et al. ${ }^{21}$ For deposited biatomic Fe chains we have also found a very good agreement between the values of the relative atomic positions obtained by relaxing a slab with nine and seven layers of the Ir substrate (see Table I).

We performed relaxations of the Fe biatomic chains only for the ferromagnetic solutions to reduce the computational effort, presenting the results in Table I. While the distance $\Delta$ between the atomic strands in the $\mathrm{C} 1$ configuration is $2.35 \AA$, and thus smaller than along the chain (see Figs. 1 


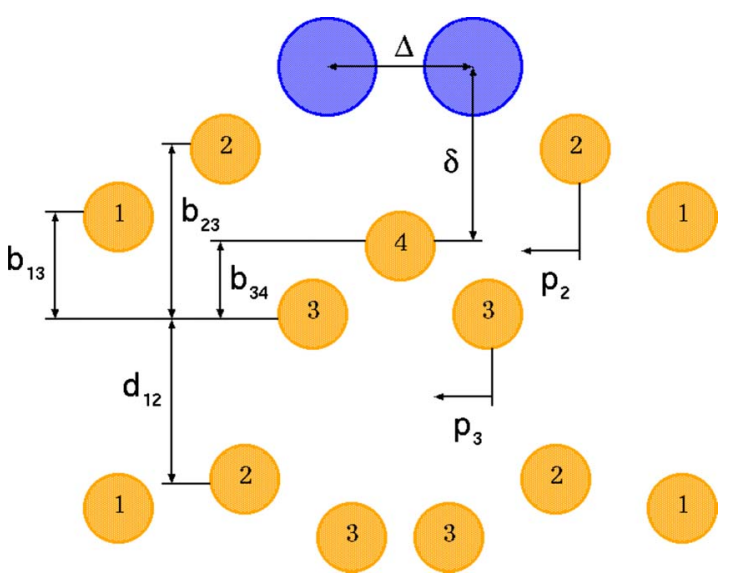

FIG. 2. (Color online) Schematical representation of the structural parameters given in Table I. Note that in this figure the buckling of the Ir substrate is greatly exaggerated and the positions of the Fe atoms (blue) and Ir atoms in the first and second layer of the substrate (gold) do not correspond to realistic calculated values.

and 2), it is almost twice larger in the $\mathrm{C} 4$ configuration, i.e., $\Delta=4.23 \AA$, and the two strands are well separated. In the latter structure, the $\mathrm{Fe}$ atoms are also more embedded into the Ir surface which is illustrated by the smaller vertical distance $\delta$ from the Ir surface atoms between the two Fe chain atoms (denoted as Ir4 in Fig. 2). The influence of the C4 chains on the buckling of the Ir substrate is also more pronounced as can be seen from the increased vertical separations of the surface Ir atoms. The different structural relaxations of the two chain configurations already hint at a larger hybridization of the Fe $3 d$ and Ir $5 d$ states and a stronger influence in the $\mathrm{C} 4$ arrangement. For both configurations the distance between the Fe dimers along the chain's axis was imposed by the Ir substrate and constituted $2.75 \AA$.

\section{MAGNETIC ORDER AND EXCHANGE INTERACTIONS}

Now we study the magnetic order and exchange interaction of the chains in the two structural arrangements. We considered the FM and AFM solutions (with antiparallel magnetic moments between adjacent $\mathrm{Fe}$ atoms along the chain) for both types of chains. For comparison, we have also calculated free-standing biatomic $\mathrm{Fe}$ chains with the same interatomic spacings as in the $\mathrm{C} 4$ and $\mathrm{C} 1$ configurations. As can be seen in Table II, the magnetic moments of the Fe atoms are slightly larger in the $\mathrm{C} 4$ configuration where the $\mathrm{Fe}$ atoms are further apart. The difference in the magnetic moments between FM and AFM state is very small. The induced moments in the Ir surface layer depend much more sensitively on the magnetic state of the Fe chains. For the FM solutions, these moments are significant in both structures and decay slightly faster for the $\mathrm{C} 1$ than for the $\mathrm{C} 4$ configuration. In the AFM state, due to symmetry some of the Ir atoms do not carry an induced moment. In the $\mathrm{C} 4$ structure, the Ir surface atom between the Fe atoms has a rather large moment of $0.18 \mu_{B}$ indicating a strong hybridization.
TABLE II. Relative total energies obtained in GGA (in meV/Fe atom), spin moments in the muffin-tin spheres of the Fe atoms in unsupported bichain (UBC) and supported bichains, as well as for Ir surface atoms, and total moments in the unit cell (in $\mu_{B}$ ) for calculations with nine and seven layers of the $(5 \times 1)$ reconstructed $\operatorname{Ir}(001)$ substrate. The Ir surface atoms are denoted as in Fig. 2.

\begin{tabular}{lcccc}
\hline \hline & C1-FM & C1-AFM & C4-FM & C4-AFM \\
\hline UBC & 3.12 & 3.14 & 3.28 & 3.22 \\
Energy & 0 & +74.1 & +751.1 & +914.6
\end{tabular}

Nine layers

\begin{tabular}{lrrrl}
\hline Energy & +9.8 & +30.9 & +33.7 & \multicolumn{1}{l}{0} \\
Total & 3.21 & 0.00 & 3.41 & 0.00 \\
Fe & 2.97 & 2.98 & 3.01 & 3.04 \\
Ir1 & 0.00 & 0.01 & 0.00 & 0.00 \\
Ir2 & -0.02 & 0.00 & 0.07 & 0.06 \\
Ir3 & 0.08 & 0.06 & 0.14 & 0.00 \\
Ir4 & 0.29 & 0.00 & 0.16 & 0.18
\end{tabular}

Seven layers

\begin{tabular}{lrrrc}
\hline Energy & 0 & +20.6 & +48.8 & +8.5 \\
Total & 3.23 & 0.00 & 3.34 & 0.00 \\
Fe & 2.98 & 2.98 & 3.01 & 3.04 \\
Ir1 & 0.00 & 0.00 & 0.00 & 0.00 \\
Ir2 & -0.03 & 0.00 & 0.08 & 0.05 \\
Ir3 & 0.08 & 0.06 & 0.14 & 0.00 \\
Ir4 & 0.29 & 0.00 & 0.15 & 0.18 \\
\hline \hline
\end{tabular}

The total energy differences between the two magnetic configurations given in Table II reveal a surprising result. In particular, we find that the preferred magnetic state is FM for the Fe chains in the $\mathrm{C} 1$ structure $\left(d_{\mathrm{Fe}-\mathrm{Fe}}=2.35 \AA\right)$, while the total energy difference is in favor of the AFM solution for the $\mathrm{C} 4$ structure with $\mathrm{Fe}$ atoms further apart $\left(d_{\mathrm{Fe}-\mathrm{Fe}}=4.23 \AA\right)$. For free-standing biatomic $\mathrm{Fe}$ wires with the same spacing between Fe atoms, the energy difference between FM and AFM states is 74 and $164 \mathrm{meV} / \mathrm{Fe}$ in favor of the FM state for the $\mathrm{C} 1$ and $\mathrm{C} 4$ configurations, respectively. For supported chains the corresponding energy differences are of the order of rather small $30 \mathrm{meV} / \mathrm{Fe}$, indicating a strong influence of the Ir substrate on the magnetic coupling and weakening of the FM interaction in the freestanding chains due to hybridization with the Ir atoms. ${ }^{30} \mathrm{Be}-$ cause of the large separation of the two strands of the biatomic chain in the $\mathrm{C} 4$ configuration, this effect is dramatic and leads to the AFM ground state. This notion is further supported by the magnitude of the exchange interaction between the two strands. We have calculated the total energy difference between a FM and a AFM alignment of the two strands of the deposited $\mathrm{Fe}$ chains which is 166 and 4 $\mathrm{meV} / \mathrm{Fe}$ in favor of ferromagnetic coupling in the $\mathrm{C} 1$ and $\mathrm{C} 4$ configurations, respectively. The extremely small value in the $\mathrm{C} 4$ configuration, indicating that the two strands are magnetically nearly decoupled, is probably due to a small indi- 


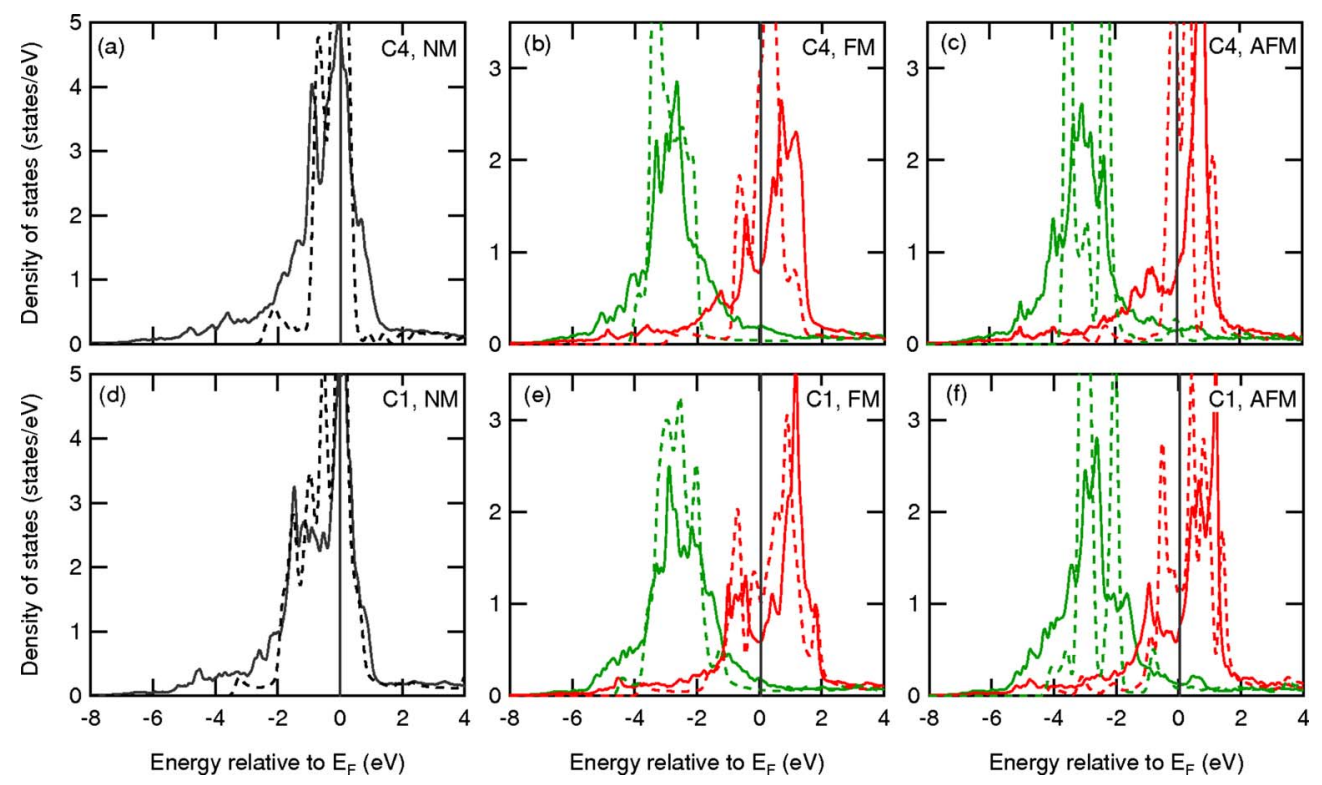

FIG. 3. (Color online) DOS for the NM, FM, and AFM states of Fe biatomic chains on nine layers of the $(5 \times 1)$ reconstructed $\operatorname{Ir}(001)$ surface in (a)-(c) C4 and (d)-(f) C1 configurations. The DOS is given inside the muffin-tin spheres of Fe atoms. The dashed lines show the DOS for unsupported Fe biatomic chains with corresponding spacings between the Fe atoms in the chain. In (b), (c), (e), and (f) left (green) and right (red) curves stand for spin-up and spin-down channels, respectively.

rect exchange interaction via the Ir substrate.

We have also calculated the FM and AFM total energy differences for both $\mathrm{C} 1$ and $\mathrm{C} 4$ configurations using seven layers of Ir substrate, and the results are compared in Table II to those obtained with nine layers. In the $\mathrm{C} 1$ structure the FM-AFM energy difference of $20.6 \mathrm{meV} / \mathrm{Fe}$ is in good agreement with the value of $21.1 \mathrm{meV} / \mathrm{Fe}$ for nine layers of substrate. In the $\mathrm{C} 4$ configuration, on the other hand, the energy difference between the AFM ground state and the FM state increases by only $7 \mathrm{meV} / \mathrm{Fe}$ for the thinner substrate. These results show that the favorable magnetic state within each structural arrangement is independent of the substrate thickness. However, in case of nine layers the C4-AFM state is lower in energy than the C1-FM solution by $9.8 \mathrm{meV} / \mathrm{Fe}$, while in case of seven layers this energy difference reverses sign and constitutes $8.5 \mathrm{meV} / \mathrm{Fe}$ in favor of the C1-FM state (see Table II). This discrepancy probably arises due to quantum well states in the Ir substrate which do not influence the total energy difference between different magnetic states within the same structural arrangement. Overall, our calculations reveal that, judging only from this energy difference, both configurations might appear in experiment and can be observed via, e.g., scanning tunneling microscopy measurements.

We have further checked the influence of the exchangecorrelation potential on the FM-AFM energy differences and found very similar results within the local-density approximation LDA. Using the LDA, a seven-layer $(5 \times 1) \operatorname{Ir}(001)$ substrate and the relaxed atomic positions found with GGA, in the $\mathrm{C} 1$ configuration the FM state is by $25.1 \mathrm{meV} / \mathrm{Fe}$ lower in energy than the AFM state (cf. a value of 20.6 $\mathrm{meV} / \mathrm{Fe}$ in GGA). In the C4 structure the AFM state is by $23.7 \mathrm{meV} / \mathrm{Fe}$ lower than the FM state (cf. a value of 40.3 $\mathrm{meV} / \mathrm{Fe}$ in GGA).

Within GGA, we also performed calculations of a collinear magnetic state with a spin arrangement of $\uparrow \downarrow \uparrow$ (periodi- cally repeated along the chain axis) for the $\mathrm{Fe}$ biatomic chains on seven layers of Ir substrate in the C1 and C4 structures. Our calculations reveal that the $\mathrm{C} 4-\uparrow \downarrow \uparrow$ state is by $16.0 \mathrm{meV} / \mathrm{Fe}$ higher in energy than the C4-AFM state and by $24.3 \mathrm{meV} / \mathrm{Fe}$ lower in energy than the C4-FM state. In the $\mathrm{C} 1$ configuration, the $\uparrow \downarrow \uparrow$ state is $9.4 \mathrm{meV} / \mathrm{Fe}$ higher in energy than the FM state, and by $11.2 \mathrm{meV} / \mathrm{Fe}$ lower in energy than the AFM state. From these three collinear magnetic solutions we can estimate the nearest-neighbor and nextnearest-neighbor exchange constants of an effective 1D Heisenberg model to be $J_{1}=-10 \mathrm{meV}$ and $J_{2}=+1 \mathrm{meV}$ for the C4 structure and $J_{1}=+5 \mathrm{meV}$ and $J_{2}=-1.3 \mathrm{meV}$ for the C1 structure. These values illustrate the strong tendency toward antiferromagnetic coupling due to the Ir substrate. Even in the $\mathrm{C} 1$ configuration, the FM nearest-neighbor coupling has become very weak. Note that a similar influence of the Ir surface has recently been reported for Fe monolayers on $\operatorname{Ir}(111)$ and for other $4 d / 5 d$ substrates. ${ }^{31}$

In order to understand the sensitive dependence of the magnetic coupling in the Fe chains upon the structural arrangement we take a look at the density of states (DOS) for the two configurations, shown in Fig. 3 in comparison with the free-standing chains. In the nonmagnetic state, the DOS of the supported Fe chains displays a large peak at the Fermi energy for both structures, however, the $d$-band width is smaller in the $\mathrm{C} 4$ structure due to the larger separation and weaker hybridization between the Fe atoms - an effect even more pronounced in free-standing wires.

In the $\mathrm{C} 1$ structure, the direct interaction between the $\mathrm{Fe}$ atoms perpendicular to the chain is much stronger than in $\mathrm{C} 4$ wires and the free-standing chains are a better approximation. Correspondingly, the FM DOS of the free-standing chains in $\mathrm{C} 1$ geometry is very similar to the supported chains, while larger changes are visible in the C4-FM configuration. This becomes even more evident from the com- 


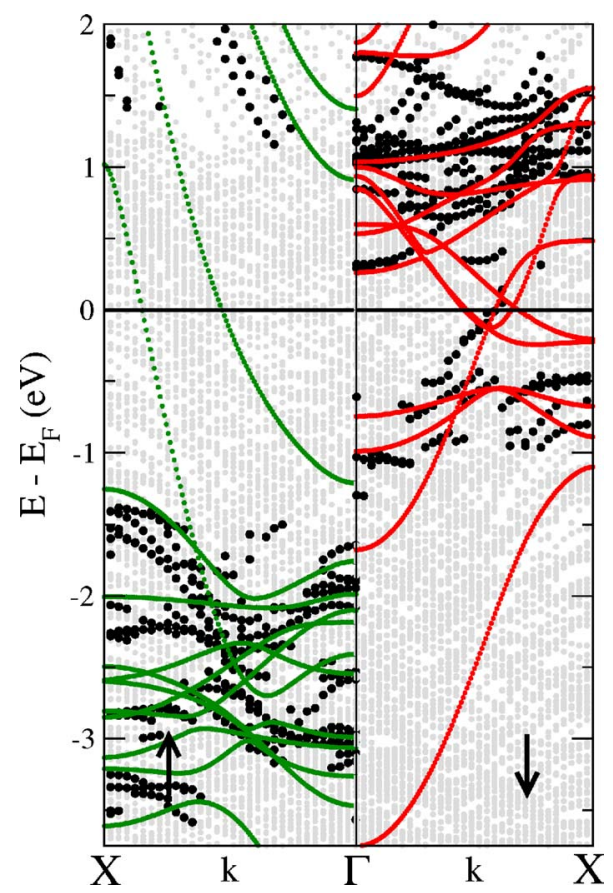

FIG. 4. (Color online) Bandstructure of the Fe biatomic chains in the $\mathrm{C} 1$-FM configuration on nine layers of the $(5 \times 1)$ reconstructed $\operatorname{Ir}(001)$ surface. Left panel shows majority bands and right panel the minority bands. $k$ vector has been chosen along the chain direction. Large black filled circles denote states which are localized on the Fe chain. For comparison the bands for an unsupported biatomic Fe chain with the same interatomic spacing are given by green (majority) and red (minority) filled small circles. Grey circles on the background mark the states of the whole $\mathrm{Fe}+\mathrm{Ir}$ system.

parison of the bandstructures of the C1-FM free-standing and C1-FM supported biatomic Fe chains presented in Fig. 4. In this plot the electronic states of the free-standing $\mathrm{C} 1$ chain (small red and small green circles) display a close correspondence to the states of the $\mathrm{C} 1$ supported chain (large black circles) which are localized mainly inside the muffin-tin spheres of Fe atoms. Remarkably, for many of the bands of the two systems a direct correspondence in terms of symmetry can be made.

For the AFM solution in both structural configurations the modifications in the DOS due to interaction with the substrate are quite significant. The electronic bands in freestanding AFM chains are normally very flat and the corresponding peaks in the DOS are very sharp. ${ }^{30}$ In this case the effect of the hybridization of the localized $3 d$-orbitals with extended states of Ir atoms on the DOS can be very strong. For both FM and AFM states, a slightly larger exchange splitting can be observed in $\mathrm{C} 4$ deposited chains, as compared to the $\mathrm{C} 1$ configuration, which leads also to larger spin moments of $\mathrm{Fe}$ atoms in the $\mathrm{C} 4$ arrangement (cf. Table II). Overall, an interplay of decreasing Fe-Fe hybridization with increasing $\mathrm{Fe}-\mathrm{Ir}$ hybridization when going from $\mathrm{C} 1$ to $\mathrm{C} 4$ structural configuration leads to somewhat larger localization of $\mathrm{Fe}$ electronic states in $\mathrm{C} 4$ biatomic chains.

\section{MAGNETOCRYSTALLINE ANISOTROPY}

As mentioned in the introduction, the magnetocrystalline anisotropy energy (MAE) is a key quantity for nanoscale magnets as it determines the preferred direction of the magnetic moments and is crucial to stabilize the magnetic order against thermal fluctuations. We have calculated the MAE for the Fe chains within the GGA employing the force theorem and found that its value is stable against the chosen ground state upon which we perform the perturbation. For the $\mathrm{C} 1$ structure, we considered the FM ground state and started from a converged ground state with an out-of-plane magnetization from which the MAE was obtained by applying the force theorem for three possible high-symmetry directions: perpendicular to the surface and the chain axis (along the $z$ axis), parallel to the axis of the chain (\| direction) and in the surface plane perpendicular to the chain's axis ( $\perp$ direction). We define two principal energies: $\mathrm{MAE}_{\|}=E_{\text {tot }}(\|)-E_{\text {tot }}(z)$, and $\mathrm{MAE}_{\perp}=E_{\text {tot }}(\perp)-E_{\text {tot }}(z)$. The number of $k$ points used for calculations constituted 144 in the full 2D-BZ. We carefully tested the MAE values with respect to the number of $k$ points. Using $144 k$-points results in an accuracy of not less than $0.3 \mathrm{meV} / \mathrm{Fe}$. We find that in the $\mathrm{C} 1-\mathrm{FM}$ configuration the calculated values are 2.1 $\mathrm{meV} / \mathrm{Fe}$ for $\mathrm{MAE}_{\|}$and $1.8 \mathrm{meV} / \mathrm{Fe}$ for $\mathrm{MAE}_{\perp}$ which corresponds to a magnetization along the $z$-axis in the ground state, i.e., perpendicular to the surface and chain axis [see Fig. 5(a)].

For the calculations of the MAE in the C4-AFM configuration we used a slab of seven layers of the $(5 \times 1) \operatorname{Ir}(001)$ substrate with $8 \mathrm{Fe}$ chain atoms in the unit cell and $144 k$ points in the full 2D-BZ (again the stability of the MAE with respect to the number of $k$-points was carefully checked). We obtain values of $-0.2 \mathrm{meV} / \mathrm{Fe}$ for $\mathrm{MAE}_{\|}$and $1.2 \mathrm{meV} / \mathrm{Fe}$ for $\mathrm{MAE}_{\perp}$ corresponding to a ground state with the magnetic moments along the chain axis [see Fig. 5(b)]. Considering the accuracy of our calculations we conclude that the easy axis of the magnetization for the Fe biatomic chains in the C4-AFM configuration constitutes a plane which cuts through the chain axis and is perpendicular to the substrate.

The MAE and its dependence on the configuration can be qualitatively related to the anisotropy of the orbital moments in the system. ${ }^{32}$ For this purpose for different magnetization directions we compare the orbital moments inside the atomic spheres of Fe chain atoms, $\mu_{L}^{\mathrm{Fe}}$, of the Ir atoms in the surface layer, $\mu_{L}^{\mathrm{Ir}}$, and the total orbital moment per unit cell, $\mu_{L}^{\text {tot }}$, defined as the sum over the moments of the two Fe chain atoms and the five Ir surface atoms on one side of the slab. For the evaluation of $\mu_{L}^{\text {tot }}$ we do not take into account the much smaller contributions from Ir atoms deeper inside the slab. For the antiferromagnetic ground state in the $\mathrm{C} 4$ geometry, one should note that the sign of the orbital moments switches for atoms of antiparallel magnetization. Therefore, we define $\mu_{L}^{\text {tot }}$ in this case as the orbital moment summed over atoms in one half of the $(2 \times 5)$ unit cell on one side of the slab.

In the C1-FM state the anisotropy of $\mu_{L}^{\text {tot }}$ is in qualitative agreement with the anisotropy of the total energy: while for an out-of-plane magnetization $\mu_{L}^{\text {tot }}$ reaches a value of $0.126 \mu_{B}$, it constitutes only $0.083 \mu_{B}$ and $0.035 \mu_{B}$ for the $\perp$ and $\|$ directions of the magnetization, respectively. Therefore, the easy axis coincides with that along which the orbital moment is largest. ${ }^{32}$ This anisotropy of $\mu_{L}^{\text {tot }}$ can be explained based on the dependence of the Ir contributions on the mag- 

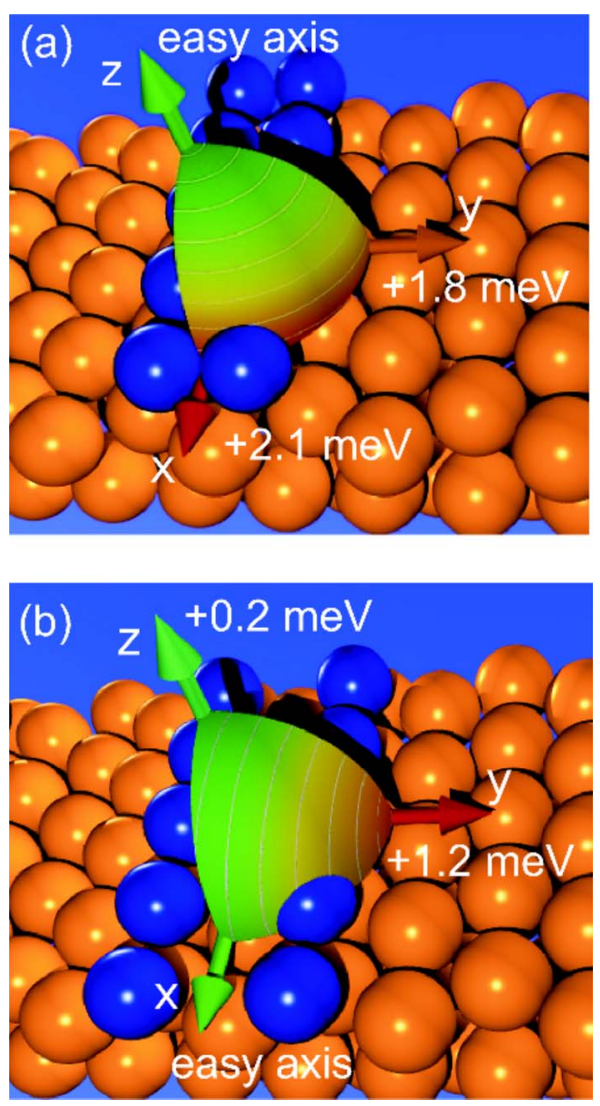

FIG. 5. (Color online) Sketches of the energy landscape as a function of magnetization direction for the two types of chains. (a) C1-FM configuration with an easy axis pointing perpendicular to the surface, hard axis along the chain $(+2.1 \mathrm{meV} / \mathrm{Fe}$ atom $)$, and middle axis perpendicular to the chain and in the surface plane (+1.8 $\mathrm{meV} / \mathrm{Fe}$ atom). (b) C4-AFM configuration with an easy axis along the chain axis but only a small energy difference with respect to the middle axis perpendicular to the surface $(+0.2 \mathrm{meV} / \mathrm{Fe}$ atom $)$, and a hard axis perpendicular to the chain and in the surface plane $(+1.2 \mathrm{meV} / \mathrm{Fe}$ atom $)$. In this plot, we construct the energy landscape based on the lowest power of the directional cosines of the magnetization with respect to the crystallographic axes which are allowed by symmetry. The coefficients are obtained from the values of $\mathrm{MAE}_{\|}$and $\mathrm{MAE}_{\perp}$.

netization direction. For an out-of-plane magnetization, the values of $\mu_{L}^{\mathrm{Ir}}$ are small and the total orbital moment is dominated by the $\mathrm{Fe}$ atoms. In contrast, for a magnetization along the $\perp$ and the $\|$ directions the Ir orbital moments, $\mu_{L}^{\mathrm{Ir}}$, reach significant values, however, of opposite sign with respect to $\mu_{L}^{\mathrm{Fe}}$. For the $\perp$-magnetization direction with $\mu_{L}^{\mathrm{Fe}}=0.077 \mu_{B}$ the value of the orbital moment of the Ir4 atom (see Fig. 2) is $-0.013 \mu_{B}$, while for the $\|$-magnetization it even reaches $-0.029 \mu_{B}$ and the corresponding value of $\mu_{L}^{\mathrm{Fe}}$ is only $0.058 \mu_{B}$.

In the C4-AFM configuration of the Fe chains the agreement between the anisotropy of the orbital moment and of the total energy is even better. While for the $\perp$-magnetization direction the value of $\mu_{L}^{\text {tot }}$ is $0.160 \mu_{B}$, it reaches much larger values of $0.239 \mu_{B}$ and $0.253 \mu_{B}$ for the $z$ and $\|$ directions, respectively. The direction of the smallest total orbital moment, $\mu_{L}^{\text {tot }}$, coincides with the hard axis and, moreover, a very small difference between the values of $\mu_{L}^{\text {tot }}$ for the two other directions corresponds to a very small energy difference $\mathrm{MAE}_{\|}$. In this structural arrangement the anisotropy of the Fe orbital moments, $\mu_{L}^{\mathrm{Fe}}$, dominates the anisotropy of $\mu_{L}^{\text {tot }}$. The contribution of the Ir atoms to the total orbital moment is nearly independent of the magnetization direction. For the $z$ - and $\|$-magnetization directions $\mu_{L}^{\mathrm{Fe}}$ is $0.100 \mu_{B}$ and $0.106 \mu_{B}$, respectively, while it is only $0.060 \mu_{B}$ and thus much smaller for the hard $\perp$-magnetization axis.

\section{SIMULATION OF STM EXPERIMENTS}

In the previous sections, we have demonstrated that the magnetic properties of biatomic Fe chains on the $(5 \times 1)$ $\operatorname{Ir}(001)$ surface depend crucially on the atomic arrangement of the atoms. Both easy magnetization direction and magnetic order change upon displacements of the $\mathrm{Fe}$ atoms which changes the interaction between the two strands of the chain and their hybridization with the Ir substrate. In order to verify our predictions experimentally, a technique with a high lateral resolution seems indispensable. Therefore, we study theoretically the possibility to use SP-STM to resolve the atomic and magnetic structure of these chains.

Figure 6 displays the calculated local density of states (LDOS) in the vacuum at a distance of about $6.8 \AA$ from the Fe chain atoms. Within the Tersoff-Hamann model of STM the vacuum LDOS is directly comparable with measured $d I / d U$ tunnel spectra. The comparison of different magnetic states shows distinct features in the FM solution for both chain structures. Strong peaks appear in the minority-spin channel at $+0.7 \mathrm{eV}$ and $+0.4 \mathrm{eV}$ in the $\mathrm{C} 4$ and $\mathrm{C} 1$ configurations, respectively [see Figs. 6(c) and 6(d)]. In the AFM state, we observe a structure of two broad peaks at $-0.5 \mathrm{eV}$ and $+1.0 \mathrm{eV}$ for the $\mathrm{C} 1$ chains, Fig. 6(f), while the $\mathrm{C} 4$ chains display a relatively featureless structure with a small peak at the Fermi energy. Considering the two favorable C1-FM and C4-AFM states of the chains, it seems that the two types can be distinguished by the strong peak of the C1-FM configuration.

A direct way of verifying the magnetic structure of the $\mathrm{Fe}$ chains might be feasible by imaging them in the topography mode of SP-STM. In addition, the structural arrangement of the atoms might be detectable. Our simulations of STM and SP-STM images are displayed in Figs. 7 and 8 for the FM ground state of the $\mathrm{C} 1$ chains and the AFM ground state of the $\mathrm{C} 4$ chains, respectively. We have chosen an energy window corresponding to the unoccupied states close to the Fermi energy, but the occupied states lead to very similar results.

The two strands of the biatomic Fe chains in the $\mathrm{C} 1$ structure are only $2.35 \AA$ apart which makes it impossible to resolve them in cross-sectional scans as seen in Figs. 7(a) and 7(b) for both spin channels and also in their summation. The corrugation amplitudes which we calculate from these plots are 1.9 and $2.5 \AA$ and the apparent width (full width at half maximum using the topmost lines of constant charge density) amounts to 7.7 and $6.9 \AA$ for the majority- and minority-spin channels, respectively. Interestingly, the chains appear wider if majority states of the chain are imaged than 

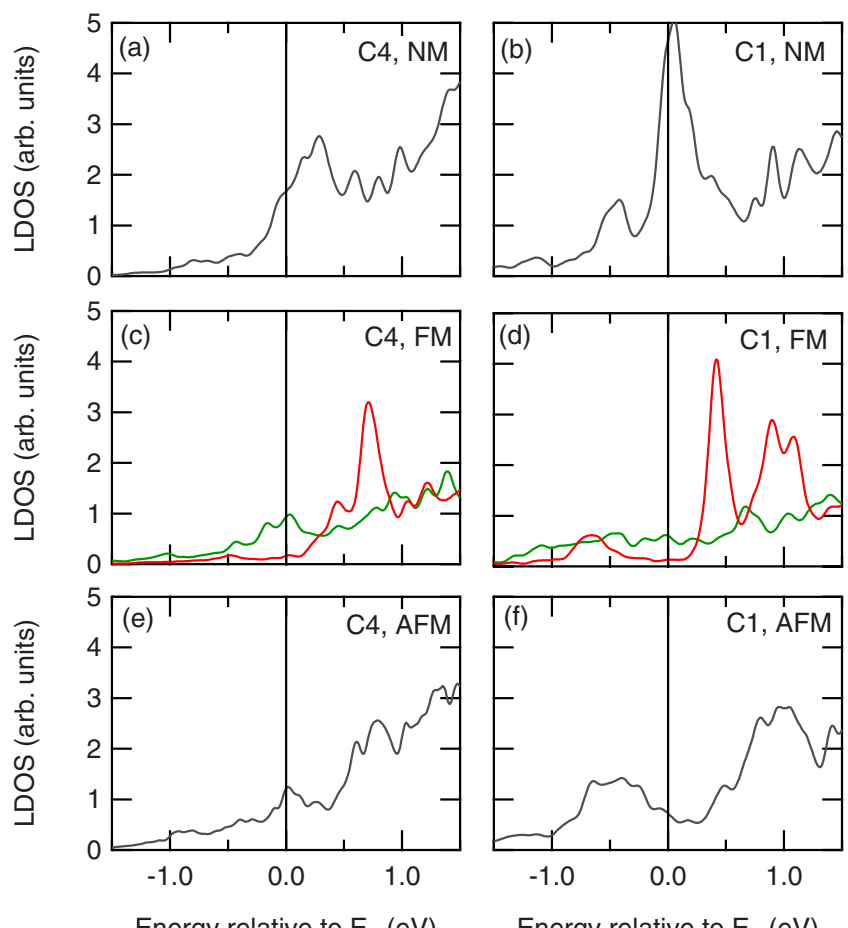

Energy relative to $E_{F}(e V)$

FIG. 6. (Color online) LDOS in the vacuum for the NM, FM, and AFM states of $\mathrm{Fe}$ biatomic chains on nine layers of the $(5 \times 1)$ reconstructed $\operatorname{Ir}(001)$ surface in $\mathrm{C} 4$ (left column) and $\mathrm{C} 1$ (right column) configurations. The LDOS is given at a distance of $\approx 6.8 \AA$ above the Fe atoms. In (c) and (d) a clear peak structure can be seen for spin-down states (red line), while the LDOS for spin-up states (green line) is featureless around the Fermi energy.

for the minority states which can be explained based on the orbital character of the dominating states. From the DOS of the Fe atoms, Fig. 3(e), we see that the minority channel is dominated by $d$-electrons while the majority $d$-band is far below the Fermi energy and therefore, $s$ - and $p$-states provide a large contribution. The $d$-character of the states for spin-down electrons is clearly visible in the cross-sectional plot, Fig. 7(b), and leads to a sharper image of the chains. The more delocalized $s$ - and $p$-states dominate the majorityspin channel, Fig. 7(a), and lead to a larger apparent width and smaller corrugation amplitude of the chains.

In the $\mathrm{C} 4$ configuration, the $\mathrm{Fe}$ chain atoms are much further apart in the perpendicular direction, $d=4.23 \AA$, which is large enough to allow the resolution of the two strands as can be seen in our simulations of STM images, shown in Fig. 8. The corrugation amplitude of the C4-AFM chains in both spin channels is 1.5 and $2.2 \AA$ in the spin-up and spin-down channels, respectively, the corrugation between the two Fe chain atoms is 0.15 and $0.3 \AA$, and the apparent widths are about $10.5 \AA$, i.e., significantly wider than the C1 FM chains. With a spin-polarized STM it should further be possible to resolve the antiferromagnetic spin alignment along the chains as can be seen from the STM images for the two separate spin channels as seen from Figs. $8(\mathrm{c})$ and $8(\mathrm{~d})$. For a spin polarization of the tip of $P_{t}=\left(n_{\uparrow}-n_{\downarrow}\right) /\left(n_{\uparrow}+n_{\downarrow}\right)=0.4$, where $n_{\uparrow}$ and $n_{\downarrow}$ are the majority and minority spin LDOS of the tip at the Fermi energy, we
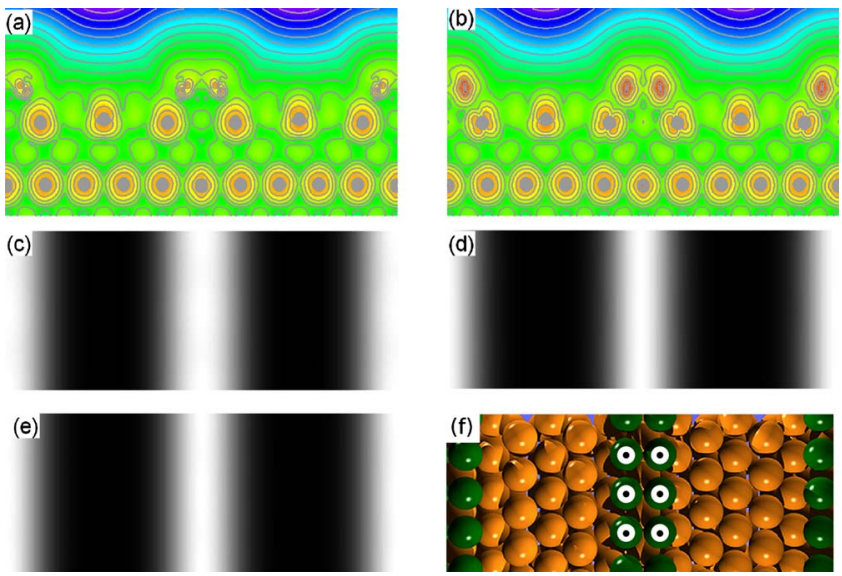

FIG. 7. (Color online) Partial charge-density plots for the FM ground state of the $\mathrm{C} 1$ structure (with seven layers of Ir substrate) with an out-of-plane easy magnetization axis in an energy regime of $\left(E_{F}, E_{F}+0.5\right) \mathrm{eV}$. (a) shows a cross-section plot of the majority states and (b) the minority states up to a distance of $5 \AA$ from the $\mathrm{Fe}$ chains (cf. Fig. 1). (a) and (b) cut through the middle green Fe atoms in (f). STM images at a distance of $5 \AA$ above the Fe chains are shown in (c) for majority electrons, (d) for minority electrons, and in (e) for the sum of both contributions. The part of the surface displayed in the STM images is given in (f). Note that the chargedensity plots are very similar for both spin directions, with the width of the charge density around the $\mathrm{Fe}$ atoms somewhat smaller for minority electrons.
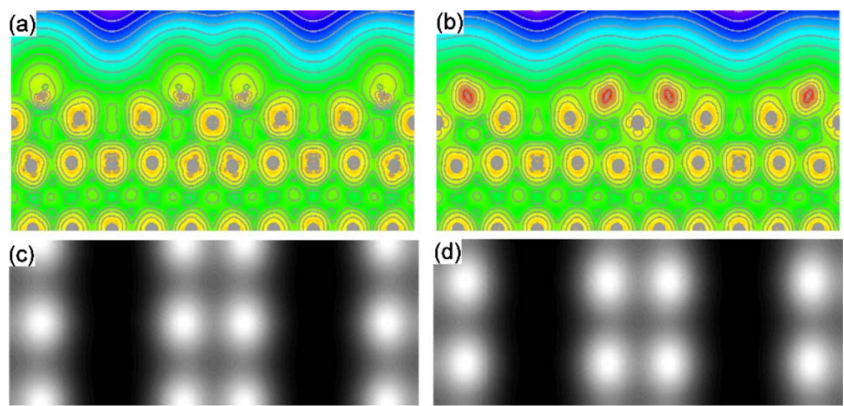

(e)
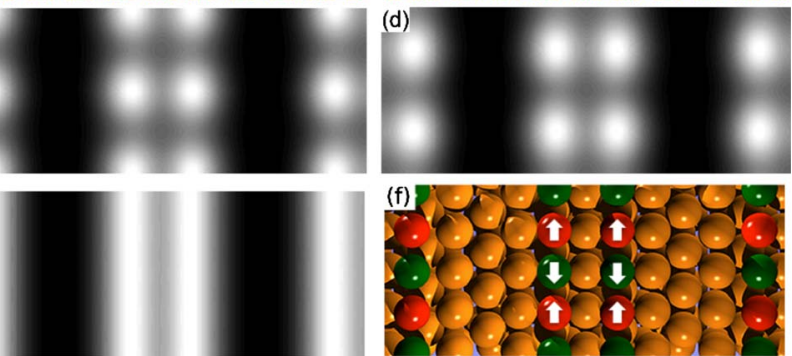

FIG. 8. (Color online) Partial charge-density plots for the AFM ground state of the $\mathrm{C} 4$ structure (with seven layers of Ir substrate) with an easy magnetization axis along the chains in an energy regime of $\left(E_{F}, E_{F}+0.3\right) \mathrm{eV}$. (a) shows a cross-section plot of the majority states and (b) the minority states up to a distance of $5 \AA$ from the Fe chains (cf. Fig. 1). (a) and (b) cut through the middle green $\mathrm{Fe}$ atoms in (f) and majority and minority states are defined with respect to these $\mathrm{Fe}$ atoms. STM images at a distance of $5 \AA$ above the Fe chains are shown in (c) for majority electrons, (d) for minority electrons, and in (e) for the sum of both contributions. The part of the surface displayed in the STM images is given in (f) where arrows indicate the direction of the Fe spin moments. Alternating color of Fe chain atoms and arrows are introduced to emphasize opposite direction of $\mathrm{Fe}$ spin moments. Note, that the spinaveraged local DOS of the two Fe atoms with opposite spin moments is identical which leads to the stripe pattern in (e). 
obtain a corrugation amplitude of $\Delta z=0.15 \AA$ along the chain, i.e., the maximum height change as the tip scans along the chain.

\section{CONCLUSIONS}

We performed extensive first-principles calculations to elucidate the interplay of structure and magnetism in biatomic $\mathrm{Fe}$ chains on the $(5 \times 1)$ reconstructed $\operatorname{Ir}(001)$ surface. We find a crucial influence of the hybridization of $\mathrm{Fe}$ chains with the Ir substrate on the magnetic ground state of the wires. Depending on the particular structural arrangement, the magnetic ground state switches from along-thechain ferromagnetic for the $\mathrm{C} 1$ configuration with a smaller $(\approx 2.4 \AA)$ distance between the two strands, to antiferromagnetic for the $\mathrm{C} 4$ state for which this distance constitutes an almost twice larger value $(\approx 4.2 \AA)$. In the $\mathrm{C} 4$ configuration, the two strands of the chain are nearly decoupled in terms of exchange interaction, while we find strong ferromagnetic coupling in the $\mathrm{C} 1$ configuration. We also find that the direction of the magnetization in these two configurations is different: while in $\mathrm{C} 1-\mathrm{FM}$ chains the $\mathrm{Fe}$ spin moments point out-of-plane with a value of the magnetic anisotropy of $\approx 2 \mathrm{meV} / \mathrm{Fe}$ with respect to in-plane directions, the magnetization in C4-AFM chains can freely rotate in the plane of along-the-chain and out-of-plane directions at sufficiently small temperatures, protected from switching to the in-plane perpendicular to the chain direction by a value of $\approx 1.2 \mathrm{meV} / \mathrm{Fe}$.

The two different magnetic types of chains are very close in total energy, which facilitates their experimental observa- tion and provides a considerable challenge for experimentalists to verify their magnetic ground state. With our calculations we provide theoretical evidence for the feasibility to use spin-polarized STM to resolve the atomic arrangement and magnetic order. Considering the rather small calculated total energy differences between the different magnetic collinear solutions of the order of $20-30 \mathrm{meV} / \mathrm{Fe}$ in both types of chains, we also cannot exclude the occurrence of noncollinear magnetic states either due to exchange interactions or due to the Dzyaloshinskii-Moriya interaction driven by spinorbit coupling. 5 ,6 Such noncollinear calculations were beyond the scope of the present work due to the large supercell required for realistic modeling of chains on the $(5 \times 1)$ reconstructed $\operatorname{Ir}(001)$ surface. However, future investigations of this system need to address this open question. Qualitatively and quantitatively different energy landscapes of the magnetization direction in real space could also result in a different response of the magnetization with respect to external magnetic field or temperature, providing an additional channel for tackling the magnetism in these two types of chains experimentally.

\section{ACKNOWLEDGMENTS}

Financial support of the Stifterverband für die Deutsche Wissenschaft and the Interdisciplinary Nanoscience Center Hamburg are gratefully acknowledged. We would like to thank Matthias Menzel, Kirsten von Bergmann, André Kubetzka, Paolo Ferriani, Gustav Bihlmayer, Stefan Blügel, and Roland Wiesendanger for many fruitful discussions.
*Corresponding author: y.mokrousov@fz-juelich.de

${ }^{1}$ G. A. Prinz, Science 282, 1660 (1998).

${ }_{2}^{2}$ S. A. Wolf, D. D. Awschalom, R. A. Buhrman, J. M. Daughton, S. von Molnár, M. L. Roukes, A. Y. Chtchelkanova, and D. M. Treger, Science 294, 1488 (2001).

${ }^{3}$ C. Chappert, A. Fert, and F. N. V. Dau, Nature Mater. 6, 813 (2007).

${ }^{4}$ C.-G. Duan, J. P. Velev, R. F. Sabirianov, Z. Zhu, J. Chu, S. S. Jaswal, and E. Y. Tsymbal, Phys. Rev. Lett. 101, 137201 (2008).

${ }^{5}$ M. Bode, M. Heide, K. von Bergmann, P. Ferriani, S. Heinze, G. Bihlmayer, A. Kubetzka, O. Pietzsch, S. Blügel, and R. Wiesendanger, Nature (London) 447, 190 (2007).

${ }^{6}$ P. Ferriani, K. von Bergmann, E. Y. Vedmedenko, S. Heinze, M. Bode, M. Heide, G. Bihlmayer, S. Blügel, and R. Wiesendanger, Phys. Rev. Lett. 101, 027201 (2008).

${ }^{7}$ C. Pfleiderer and U. K. Rössler, Nature (London) 447, 157 (2007).

${ }^{8}$ P. Ferriani, I. Turek, S. Heinze, G. Bihlmayer, and S. Blügel, Phys. Rev. Lett. 99, 187203 (2007).

${ }^{9}$ P. Gambardella, A. Dallmeyer, K. Maiti, M. C. Malagoli, W. Eberhardt, K. Kern, and C. Carbone, Nature (London) 416, 301 (2002).

${ }^{10}$ P. Gambardella, A. Dallmeyer, K. Maiti, M. C. Malagoli, S. Rusponi, P. Ohresser, W. Eberhardt, C. Carbone, and K. Kern, Phys. Rev. Lett. 93, 077203 (2004).
${ }^{11}$ C. F. Hirjibehedin, C. P. Lutz, and A. J. Heinrich, Science 312, 1021 (2006).

${ }^{12}$ J. Dorantes-Davila and G. M. Pastor, Phys. Rev. Lett. 81, 208 (1998).

${ }^{13}$ M. Komelj, C. Ederer, J. W. Davenport, and M. Fähnle, Phys. Rev. B 66, 140407(R) (2002).

${ }^{14}$ B. Újfalussy, B. Lazarovits, L. Szunyogh, G. M. Stocks, and P. Weinberger, Phys. Rev. B 70, 100404(R) (2004).

${ }^{15}$ S. Baud, Ch. Ramseyer, G. Bihlmayer, and S. Blügel, Phys. Rev. B 73, 104427 (2006).

${ }^{16}$ A. N. Rudenko, V. V. Mazurenko, V. I. Anisimov, and A. I. Lichtenstein, Phys. Rev. B 79, 144418 (2009).

${ }^{17}$ M. C. Urdaniz, M. A. Barral, and A. M. Llois, Physica B 404, 2822 (2009).

${ }^{18}$ L. Hammer, W. Meier, A. Schmidt, and K. Heinz, Phys. Rev. B 67, 125422 (2003).

${ }^{19}$ L. Hammer, W. Meier, A. Klein, P. Landfried, A. Schmidt, and K. Heinz, Phys. Rev. Lett. 91, 156101 (2003).

${ }^{20}$ A. Klein, A. Schmidt, L. Hammer, and K. Heinz, Europhys. Lett. 65, 830 (2004).

${ }^{21}$ D. Spišák and J. Hafner, Surf. Sci. 546, 27 (2003).

${ }^{22}$ K. von Bergmann, S. Heinze, M. Bode, E. Y. Vedmedenko, G. Bihlmayer, S. Blügel, and R. Wiesendanger, Phys. Rev. Lett. 96, 167203 (2006).

${ }^{23}$ A. Kubetzka, P. Ferriani, M. Bode, S. Heinze, G. Bihlmayer, K. 
von Bergmann, O. Pietzsch, S. Blügel, and R. Wiesendanger, Phys. Rev. Lett. 94, 087204 (2005).

${ }^{24}$ J. Honolka, T. Y. Lee, K. Kuhnke, D. Repetto, V. Sessi, P. Wahl, A. Buchsbaum, P. Varga, S. Gardonio, C. Carbone, S. R. Krishnakumar, P. Gambardella, M. Komelj, R. Singer, M. Fähnle, K. Fauth, G. Schütz, A. Enders, and K. Kern, Phys. Rev. B 79, 104430 (2009).

${ }^{25}$ J. Honolka, T. Y. Lee, K. Kuhnke, A. Enders, R. Skomski, S. Bornemann, S. Mankovsky, J. Minár, J. Staunton, H. Ebert, M. Hessler, K. Fauth, G. Schütz, A. Buchsbaum, M. Schmid, P. Varga, and K. Kern, Phys. Rev. Lett. 102, 067207 (2009).

${ }^{26}$ In the process of writing this paper we became aware of a recent publication by R. Mazzarello and E. Tosatti, Phys. Rev. B 79,
134402 (2009), reporting on first-principles investigation of the same system.

${ }^{27}$ Y. Zhang and W. Yang, Phys. Rev. Lett. 80, 890 (1998).

${ }^{28}$ S. H. Vosko, L. Wilk, and M. Nussair, Can. J. Phys. 58, 1200 (1980).

${ }^{29}$ D. Lerch, S. Müller, L. Hammer, and K. Heinz, Phys. Rev. B 74, 075426 (2006).

${ }^{30}$ Y. Mokrousov, G. Bihlmayer, S. Blügel, and S. Heinze, Phys. Rev. B 75, 104413 (2007).

${ }^{31}$ B. Hardrat, A. Al-Zubi, P. Ferriani, S. Blügel, G. Bihlmayer, and S. Heinze, Phys. Rev. B 79, 094411 (2009).

${ }^{32}$ P. Bruno, Phys. Rev. B 39, 865 (1989). 